\title{
An Image Restoration Method Using Matrix Transform and Gaussian Mixture Model for Radio Tomographic Imaging
}

\author{
Fei Gao, Cheng Sun, Heng Liu, Jianping An, and Shengxin Xu \\ School of Information and Electronics, Beijing Institute of Technology, Beijing, China \\ Correspondence should be addressed to Heng Liu; lhengzzt@bit.edu.cn
}

Received 17 January 2017; Revised 24 April 2017; Accepted 17 May 2017; Published 20 June 2017

Academic Editor: Pierre-Martin Tardif

Copyright (c) 2017 Fei Gao et al. This is an open access article distributed under the Creative Commons Attribution License, which permits unrestricted use, distribution, and reproduction in any medium, provided the original work is properly cited.

\begin{abstract}
Radio Tomographic Imaging (RTI) is an attractive technique for imaging the nonmetallic targets within wireless sensor network. RTI has been used in many challenging environments and situations. Due to the accuracy of Radio Tomographic Imaging system model and the interference between the wireless signals of sensors, the image obtained from the RTI system is a degraded target image, which cannot offer sufficient details to distinguish different targets. In this paper, we treat the RTI system as an image degraded process, and we propose an estimation model based on mixture Gaussian distribution to derive the degradation function from the shadowing-based RTI model. Then we use this degradation function to recover an original image by a method called constrained least squares filtering. So far, many imaging models have been proposed for localization; however, they do not have a satisfied imaging accuracy. Simulated and experimental results show that the imaging accuracy of our proposed method is improved, and the proposed method can be used in the real-time circumstances.
\end{abstract}

\section{Introduction}

Radio Tomographic Imaging (RTI) is an emerging technology for imaging the attenuation of nonmetallic targets within wireless sensor network area. The presence of targets between the transmitters and receivers leads to changes in the received signal strength (RSS) measurements at the receivers. RTI uses these changes to reconstruct an image of the propagation field. The images can provide the location of targets and their movements [1]. Therefore, RTI has been gaining great interest from a variety of applications, for example, road surveillance [2], health care [3], through-wall tracking [4], and floor plan mapping $[5,6]$.

Shadowing-based RTI (SRTI) was proposed by Wilson and Patwari [7] which was the original imaging approach by using RSS variation obtained from a wireless network. SRTI assumed that the obstructed wireless links suffer from large shadowing loss, and the RSS of the links which were not obstructed by the targets keep stable. The assumption is valid for an open environment, so SRTI is not suitable for the indoor environments where RSS varies easily due to the multipath effect. Wilson and Patwari proposed
Variance-Based RTI (VRTI) which introduced the variance of RSS to improve the tracking performance in indoor environments [4]. A fade level-based spatial model for RTI was proposed, which divided the links into two different types: deep fade links and antifade links [8]. Zhao et al. [9] have proposed kernel distance-based RTI (KRTI) which used the kernel distance between short-term and long-term RSS histograms to estimate the image of human presence. Wei et al. [10] have proposed directional RTI (dRTI) which employed electronically switched directional (ESD) antennas to mitigate the multipath effect. However, using directional antennas will increase the sensor size and the cost of radio sensors. Wang et al. [11] proposed enhanced SRTI (ESRTI) which employed the interference link canceling technique to enhance indoor RTI image quality and tracking accuracy.

In this paper, we address the target imaging issue in RTI; that is, we focus on getting the "original" undistorted target image rather than improving the locating or tracking performance of targets [12]. Previous researches have paid full attention to locating and tracking targets $[13,14]$, and these RTI methods have resulted in a dilative imaging area of the targets. The imaging result does not offer sufficient details 


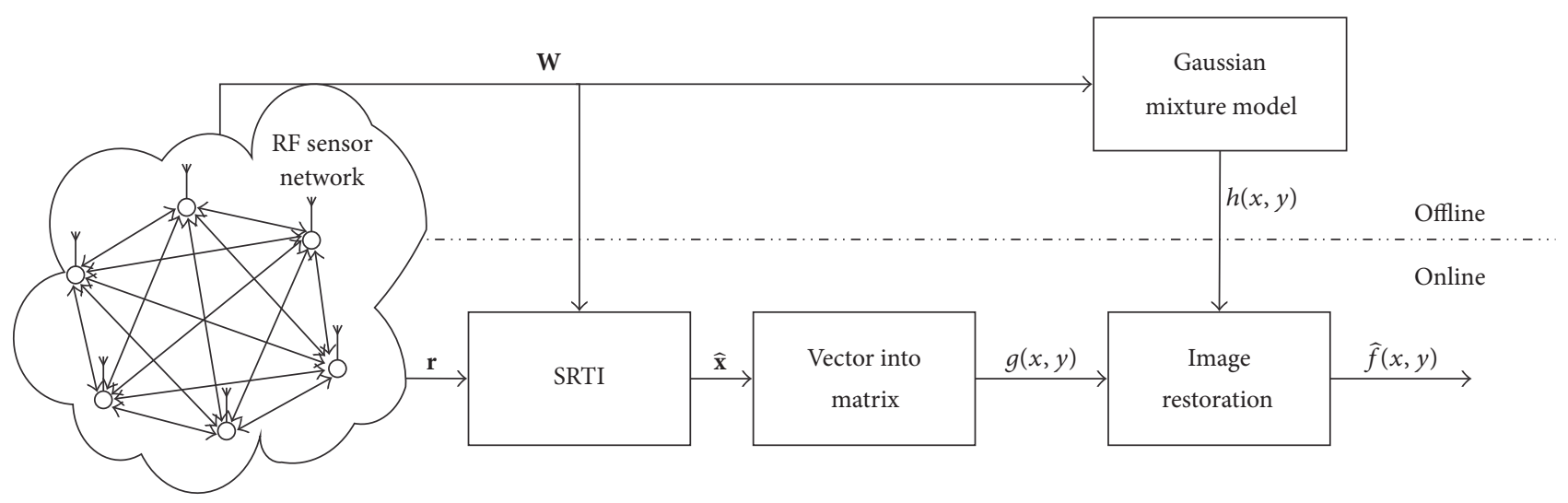

FIGURE 1: System overview of the proposed method.

to recognize the desired targets. The dilation effect is caused by the limited wireless communication links. When the links increase, the more wireless sensor nodes and the longer time of scanning all communication links will be needed. Meanwhile, the interference between the sensor nodes is enhanced which leads to a worse imaging result. Therefore, we propose the image restoration technique to solve the poor imaging problem in RTI. Image restoration is a technology that attempts to recover an "original" image from the "degraded" image by using a priori knowledge of the degradation process [15]. After a priori knowledge of the degradation process is obtained or estimated, the "original" image can be recovered by applying the inverse process. Therefore, we propose a novel technique for getting the undistorted images of targets. Our approach involves applying matrix theory and Gaussian mixture model to estimate the degradation function of RTI system, which describes the degradation phenomenon in RTI system and is outlined in Section 3. Further, we take the simulated objects and active human as the standard targets to analyze the performance of our proposed approach.

Our proposed method operates as illustrated in Figure 1. In short, the system is divided into two phases. In the offline phase, we calculate the weight matrix $\mathbf{W}$ of the wireless network; then we use Gaussian mixture model to estimate the elements of the degradation function $h(x, y)$. In the online phase, we first acquire RSS measurement changes $\mathbf{r}$ from $S$ radio frequency sensors of the wireless network. The RSS changes and weight matrix are inputted to the SRTI calculating unit to form a discretized propagation image $\widehat{\mathbf{x}}$ of the monitored area. Then 2-dimensional image $g(x, y)$ is obtained after the procedure named vector into matrix. Finally, an image restoration algorithm is used to estimate the original image, and $\widehat{f}(x, y)$ is the final output image.

The rest of this paper is organized as follows. In Section 2, we introduce some preliminaries about RTI system and image restoration process. Section 3 presents the method of estimating the degradation function of RTI system. Section 4 presents the simulated and experimental results of the proposed image restoration method in RTI system. Section 5 discusses the conclusions.

\section{Radio Tomographic Imaging and Image Restoration}

In this section, we introduce the RTI model which relates radio power attenuation in spatial voxels to the difference of RSS measured on the links of a wireless network. And a model of image degradation/restoration process is also introduced.

2.1. Radio Tomographic Imaging. When a wireless sensor network communicates through transmission of wireless signal, the RSS measurements on the receiving node of a link are affected by the targets between the sensors. Figure 2 illustrates a simple wireless network and the reconstructed image by using RSS measurements. When there are $S$ radio frequency sensors at the perimeter of the monitored region, the total number of unidirectional links is $L=S(S-1) / 2$. The variation of RSS in $\mathrm{dBm}$ on link $l$ can be written as

$$
\Delta r_{l, t}=r_{l, 0}-r_{l, t}, \quad l=1,2, \ldots, L,
$$

where $r_{l, 0}$ is the static RSS of link $l$ when there is no target in the monitored region and $r_{l, t}$ is the RSS of link $l$ at time instant $t$ when the targets appear in the monitored region.

Assume that the change in RSS is a spatial integral of the attenuation field in the monitored region. When the attenuation field is divided into voxels, the change in RSS of each link can be treated as a linear combination of the change caused by each voxel [16]:

$$
\Delta r_{l, t}=\sum_{j=1}^{N} W_{l j} \Delta x_{j, t}+n_{l, t}
$$

where $\Delta x_{j, t}$ is the RSS attenuation occuring in voxel $j$ at the time instant $t, N$ is the number of voxels in the image, $n_{l, t}$ is the measurement noise of link $l$ at time instant $t$, and $W_{l j}$ is the weight of voxel $j$ for link $l$. We use the normalized ellipse model to obtain $W_{l j}[17]$ :

$$
W_{l j}=\frac{1}{\sqrt{d_{l}}} \begin{cases}1 & \text { if } d_{l j}(1)+d_{l j}(2)<d_{l}+\lambda \\ 0 & \text { otherwise }\end{cases}
$$




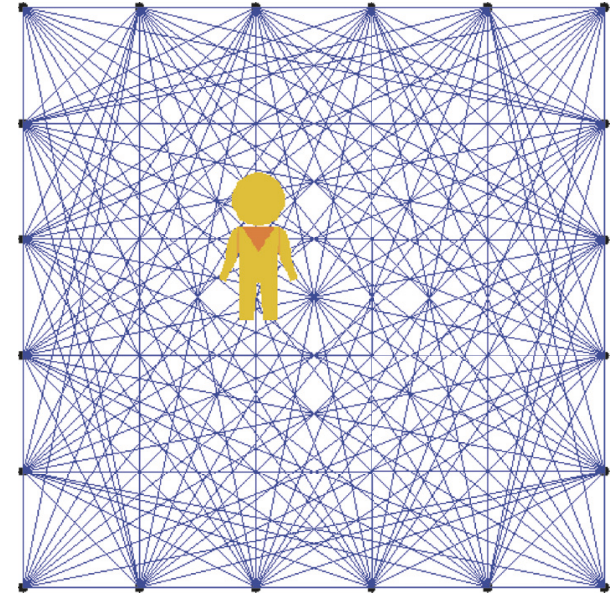

(a)

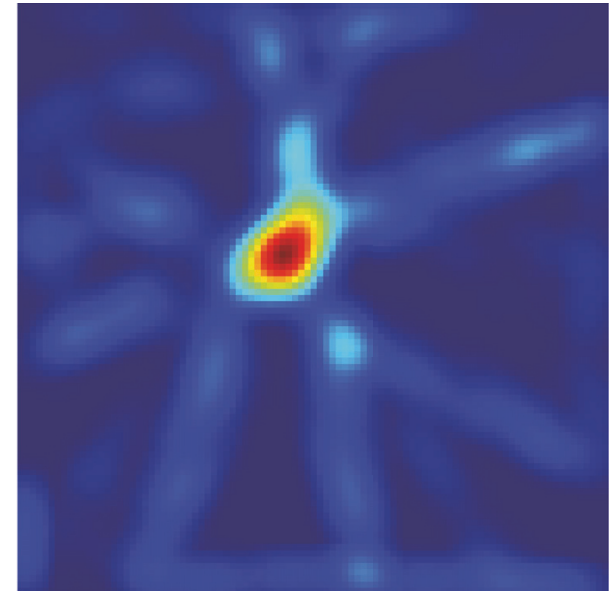

(b)

FIGURE 2: An illustration of RTI network. (a) shows a target in the propagation field of RF sensors. (b) shows the RTI reconstructed image of scene.

where $d_{l}$ is the distance between two sensors (the transmitter and the receiver) and $d_{l j}(1)$ and $d_{l j}(2)$ are the distances between voxel $j$ and the two sensors of link $l$, respectively. $\lambda$ is the parameter describing the width of the ellipse. If all links in the network are considered, (2) can be rewritten as

$$
\mathbf{r}=\mathbf{W} \mathbf{x}+\mathbf{n},
$$

where $\mathbf{r}$ and $\mathbf{n}$ represent the RSS changes and noises of $L$ wireless links, respectively, and they are column vectors of size $L \times 1 . \mathbf{x}$ is an $N \times 1$ column vector representing the attenuation image. $[\mathbf{W}]_{l j}=W_{l j}$ is an $L \times N$ matrix representing the weight matrix. This equation describes the relationship between the image of target presence and the RSS changes of wireless sensor network, which is called shadowing-based RTI.

Estimating the image $\mathbf{x}$ from the links measurements $\mathbf{r}$ is an ill-posed inverse problem, so regularization is required [18]. We use the Tikhonov regularization method to handle this ill-posedness. We can define the linear transformation matrix $\Pi$ :

$$
\boldsymbol{\Pi}=\left(\mathbf{W}^{T} \mathbf{W}+\alpha\left(\mathbf{D}_{X}^{T} \mathbf{D}_{X}+\mathbf{D}_{Y}^{T} \mathbf{D}_{Y}\right)\right)^{-1} \mathbf{W}^{T},
$$

where $\alpha$ is the regularization parameter and matrix $\mathbf{D}_{X}$ and matrix $\mathbf{D}_{Y}$ are the difference operator for the horizontal and vertical direction, respectively. Then the solution of (4) is given by

$$
\widehat{\mathbf{x}}=\Pi \mathbf{r} .
$$

In view of the accuracy of measurement and calculation, the reconstructed image has a peripheral dilation effect within the target area (as shown in Figure 2), and it could not show the small edge information of the target. Therefore the dilation effect should be eliminated by using the image restoration method.

2.2. Image Restoration. In image restoration, we consider the degradation and restoration process as a linear model, as

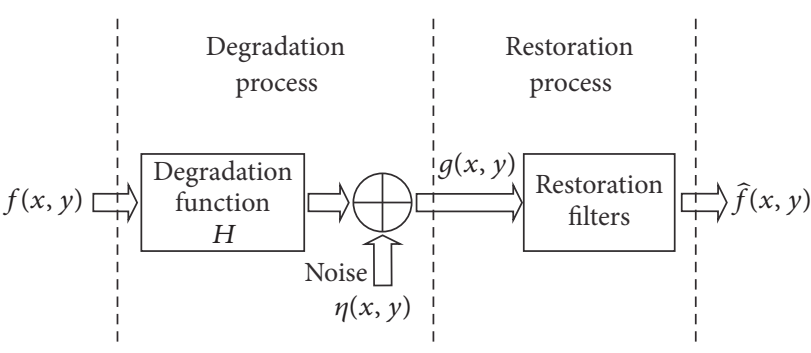

FIgURE 3: A model of the image degradation/restoration process.

shown in Figure 3. The degradation function and an additive noise term are operated on an input image to produce a degraded image [19]. Now we assume $H$ is a linear, positioninvariant blurring process; then the degraded image can be given as

$$
g(x, y)=h(x, y) * f(x, y)+\eta(x, y),
$$

where $h(x, y)$ is the degradation function in spatial domain and the symbol " *" indicates a convolution operator.

As is known, convolution in the spatial domain is analogous to multiplication in the frequency domain, so we can express (7) in the frequency domain:

$$
G(u, v)=H(u, v) F(u, v)+N(u, v),
$$

where the terms in capital letters are the Fourier transforms of the corresponding terms in (7).

Since the problem of estimating the input image $f(x, y)$ is typically ill-posed [20], in order to get the original input image $f(x, y)$, we introduce the method called constrained least squares that minimizes the criterion to smooth the image. The objective function is defined as

$$
\begin{aligned}
& C_{\gamma}(f, h)=\sum_{x, y}\left\{[g(x, y)-h(x, y) * f(x, y)]^{2}\right. \\
& \left.\quad+\gamma[l(x, y) * f(x, y)]^{2}\right\} ;
\end{aligned}
$$


the second term $l(x, y) * f(x, y)$ is a regularization function embodying the prior information about $f(x, y)$ [21]. We consider the optimality of restoration as a measure of smoothness, and we expect a high level of local correlation in the image. Therefore we choose a high pass filter (Laplacian filter) to minimize the high-frequency content in the solution. $\gamma$ is a tunable parameter.

The frequency domain solution to this optimization problem is the following expression [15]:

$$
\widehat{F}(u, v)=\frac{H^{*}(u, v)}{|H(u, v)|^{2}+\gamma|L(u, v)|^{2}} G(u, v),
$$

where $L(u, v)$ is the Fourier transform of $l(x, y)$ and $H^{*}(u, v)$ is the complex conjugate of $H(u, v)$. Then we can obtain the estimated original image by applying inverse Fourier transform on (10):

$$
\widehat{f}(x, y)=\operatorname{IDFT}(\widehat{F}(u, v)) .
$$

\section{Estimating the Degradation Function of RTI System}

From the previous discussion, the degraded image $g(x, y)$ of SRTI system can be obtained from (6), but the degradation function is unknown. In this section, we study the relationship between degradation function and linear solution of SRTI system. Then we propose a Gaussian mixture model to estimate the degradation function of SRTI system.

3.1. Relationship between Degradation Function and Linear Solution. By using the definition of convolution, we can express the image degradation process (7) in vector-matrix form $[15,22]$ :

$$
\mathbf{g}=\mathbf{H f}+\boldsymbol{\eta}
$$

where $\mathbf{f}$ is an $X Y \times 1$ vector representing an input image $f(x, y)$, and we can form the vector by using each column of image $f(x, y)$. $X$ and $Y$ are the width and height of the image, respectively. Then vectors $\mathbf{g}$ and $\boldsymbol{\eta}$ are formed in the same way. As a result, $\mathbf{H}$ is an $X Y \times X Y$ matrix, and its elements are given by the definition of circular convolution in two dimensions:

$$
f(x, y) * h(x, y)=\sum_{m=0}^{X-1} \sum_{n=0}^{Y-1} f(m, n) h(x-m, y-n) .
$$

Suppose the degradation function $h(x, y)$ has dimensions $(2 K+1) \times(2 K+1)$, and its elements are shown in the following matrix:

$$
\left(\begin{array}{cccccc}
h_{-K,-K} & h_{-K,-K+1} & \cdots & h_{-K, 0} & \cdots & h_{-K, K} \\
h_{-K+1,-K} & h_{-K+1,-K+1} & \cdots & h_{-K+1,0} & \cdots & h_{-K+1, K} \\
\vdots & \vdots & \ddots & \vdots & \ddots & \vdots \\
h_{0,-K} & h_{0,-K+1} & \cdots & h_{0,0} & \cdots & h_{0, K} \\
\vdots & \vdots & \ddots & \vdots & \ddots & \vdots \\
h_{K,-K} & h_{K,-K+1} & \cdots & h_{K, 0} & \cdots & h_{K, K}
\end{array}\right) .
$$

Then we plug (14) into (13) resulting in

H

$$
=\left(\begin{array}{cccccccc}
\mathbf{H}_{0} & \mathbf{H}_{-1} & \cdots & \mathbf{H}_{-K} & \mathbf{0} & \cdots & \mathbf{0} & \mathbf{0} \\
\mathbf{H}_{1} & \mathbf{H}_{0} & \cdots & \mathbf{H}_{-K+1} & \mathbf{H}_{-K} & \cdots & \mathbf{0} & \mathbf{0} \\
\vdots & \vdots & \ddots & \vdots & \ddots & \vdots & \vdots & \\
\mathbf{H}_{K} & \mathbf{H}_{K-1} & \cdots & \mathbf{H}_{0} & \mathbf{H}_{-1} & \cdots & \mathbf{0} & \mathbf{0} \\
\mathbf{0} & \mathbf{H}_{K} & \cdots & \mathbf{H}_{1} & \mathbf{H}_{0} & \cdots & \mathbf{0} & \mathbf{0} \\
\vdots & \vdots & \ddots & \vdots & \ddots & \vdots & \vdots & \\
\mathbf{0} & \mathbf{0} & \cdots & \mathbf{0} & \mathbf{0} & \cdots & \mathbf{H}_{0} & \mathbf{H}_{-1} \\
\mathbf{0} & \mathbf{0} & \cdots & \mathbf{0} & \mathbf{0} & \cdots & \mathbf{H}_{1} & \mathbf{H}_{0}
\end{array}\right),
$$

where $\mathbf{H}_{i}$ is a block matrix, and it is constructed by the elements of degradation function:

$$
\begin{aligned}
& \mathbf{H}_{i} \\
& =\left(\begin{array}{cccccccc}
h_{0, i} & h_{-1, i} & \cdots & h_{-K, i} & 0 & \cdots & 0 & 0 \\
h_{1, i} & h_{0, i} & \cdots & h_{-K+1, i} & h_{-K, i} & \cdots & 0 & 0 \\
\vdots & \vdots & \ddots & \vdots & \vdots & \ddots & \vdots & \vdots \\
h_{K, i} & h_{K-1, i} & \cdots & h_{0, i} & h_{-1, i} & \cdots & 0 & 0 \\
0 & h_{K, i} & \cdots & h_{1, i} & h_{0, i} & \cdots & 0 & 0 \\
\vdots & \vdots & \ddots & \vdots & \vdots & \ddots & \vdots & \vdots \\
0 & 0 & \cdots & 0 & 0 & \cdots & h_{0, i} & h_{-1, i} \\
0 & 0 & \cdots & 0 & 0 & \cdots & h_{1, i} & h_{0, i}
\end{array}\right) .
\end{aligned}
$$

As discussed before, the Tikhonov solution of SRTI system is expressed in (6); then we plug (4) into (6) which results in

$$
\widehat{\mathbf{x}}=\Pi \mathbf{r}=\Pi \mathbf{W x}+\mathbf{n}_{\mathbf{t}} .
$$

Comparing two equations (12) and (17), we find that the left sides of two equations describe the same distorted image. Suppose the noise is the same; we can get the following equation:

$$
\text { Hf }=\Pi W x \text {. }
$$

Because $\mathbf{f}$ and $\mathbf{x}$ are the same original image vectors, we can deduce that

$$
\mathbf{H}=\boldsymbol{\Pi W} \text {. }
$$

Therefore, we obtain the convolution transform matrix of SRTI system from the linear transformation matrix and the weight matrix, and the elements of degradation function are located in certain positions of convolution transform matrix.

3.2. Mixture Model of Element Estimation. In the previous subsection, we find that the elements of matrix $\mathbf{H}$ derive from the particular elements of degradation function. In 


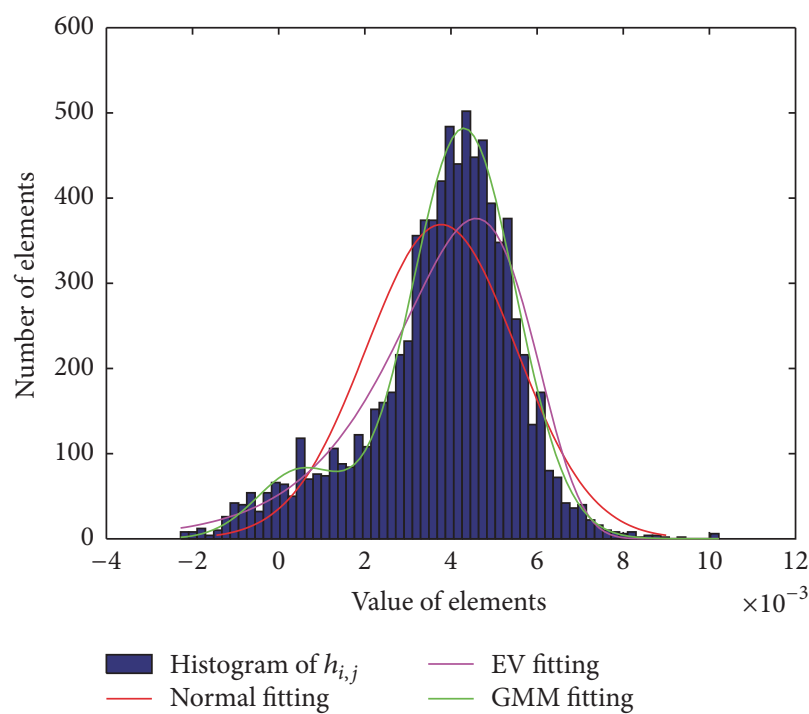

(a)

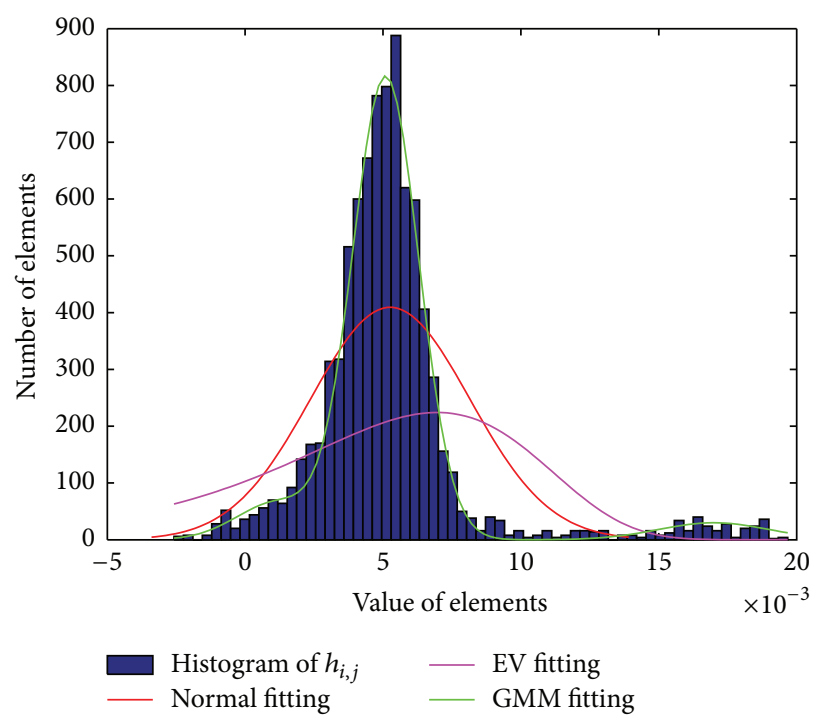

(b)

FIGURE 4: Fitting results in different probability distributions. (a) GMM with 2 Gaussian distributions in $h(5,6)$. (b) GMM with 3 Gaussian distributions in $h(13,9)$.

order to obtain the degradation function of RTI system, we consider the values of a particular element in the matrix $\mathbf{H}$ as a sampling process on the particular element in degradation function $h(x, y)$. When we analyze the distribution of the element values in the degradation function $h(x, y)$, we find that a single probability distribution cannot fit every elements in $h(x, y)$, as illustrated in Figure 4.

The fitting results show that normal distribution fitting and extreme value fitting could not fit the histogram of the element values, especially the extreme value fitting result in Figure 4(b). Gaussian mixture model could fit the histogram of the element values very well (green lines in Figure 4). The reason is that Gaussian mixture model could elect the appropriate value about the parameters of Gaussian distributions and the corresponding weighting factors to fit the histogram of the element values. Therefore we model each element in degradation function as a mixture of $Q$ Gaussian distributions:

$$
P\left(h_{i, j}\right)=\sum_{q=1}^{Q} \omega_{q} \cdot g\left(h_{i, j}, \mu_{q}, \sigma_{q}\right),
$$

where $h_{i, j}$ is the element value in degradation function $h(x, y)$ at position $(i, j), P\left(h_{i, j}\right)$ is the probability density function of $h_{i, j}, Q$ is the number of Gaussian distributions, $\omega_{q}$ is the weight of the qth Gaussian distribution, and all is $1 . g\left(h_{i, j}, \mu_{q}, \sigma_{q}\right)$ stands for the Gaussian possibility density function; $\mu_{q}$ and $\sigma_{q}$ represent its mean and standard variance, respectively.

Because the samples of each element in degradation function are located in many different positions in $\mathbf{H}$, we introduce a learning algorithm [23] to update the model parameters in time.

The first thing is assigning the elements in $\mathbf{H}$ to the corresponding group $h_{i, j, m}(1 \leq m \leq M)$ by using the mapping relationship shown in (15) and (16). Then we check each element value in the first data group. If the element value does not match the current $Q$ Gaussian distributions, we use a new Gaussian distribution to replace the least probable distribution. The mean of this new distribution is the current element value, the initial variance is a high value, and the prior weight is a low value.

When the element value matches one of the $Q$ distributions, the prior weights of the $Q$ distributions for the $n$th element are adjusted as follows:

$$
\omega_{q, m}=(1-\beta) \omega_{q, m-1}+\beta\left(M_{q, m}\right),
$$

where $\beta$ is the learning rate which defines the speed of convergence of distribution's parameters. $M_{q, m}$ is 1 for the model which is matched and 0 for the remaining models.

The parameters $\mu$ and $\sigma$ for unmatched distributions keep unaltered, and the parameters of matched distribution are updated as follows:

$$
\begin{aligned}
\mu_{m} & =(1-\rho) \mu_{m-1}+\rho h_{i, j, m}, \\
\sigma_{m}^{2} & =(1-\rho) \sigma_{m-1}^{2}+\rho\left(h_{i, j, m}-\mu_{m}\right)^{2},
\end{aligned}
$$

where $\rho=\beta g\left(h_{i, j, m} \mid \mu_{q}, \sigma_{q}\right)$ is another learning rate indicating that only the element value matching the model is included in the estimation. After all the element values in one group are processed, we calculate the corresponding degradation function element $h_{i, j}$ by

$$
\widehat{h}_{i, j}=\sum_{q=1}^{Q} \omega_{q} \cdot \mu_{q} .
$$

As each element in $h(x, y)$ is processed, the degradation function is obtained. Then we can use (10) to estimate the original RTI image. 

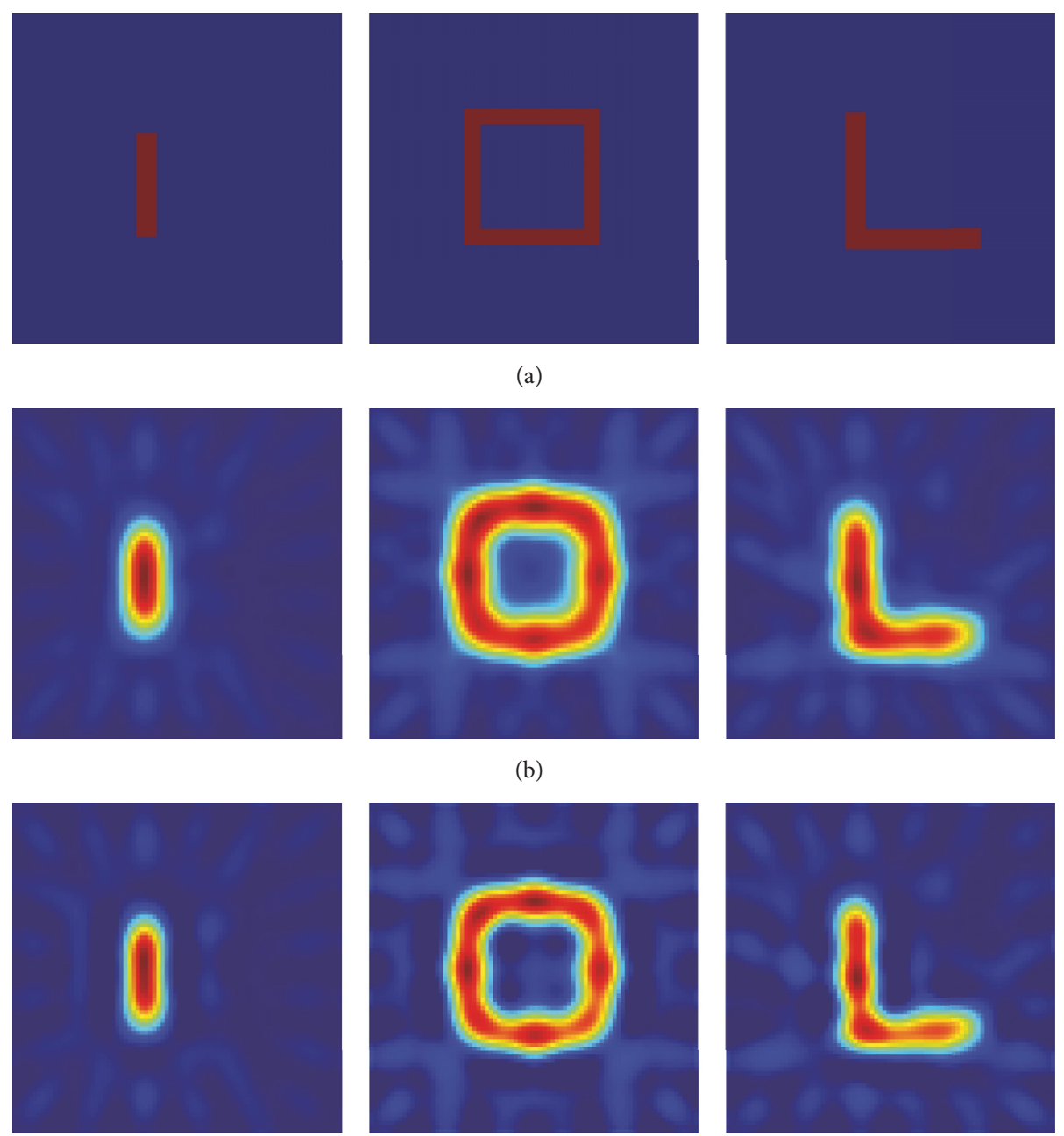

(a)
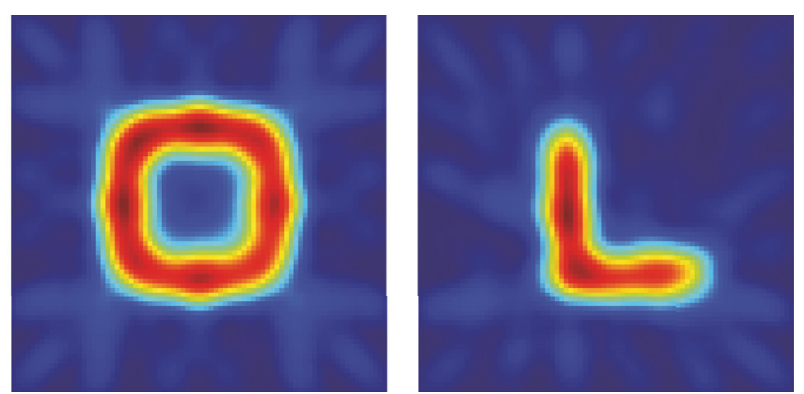

(b)
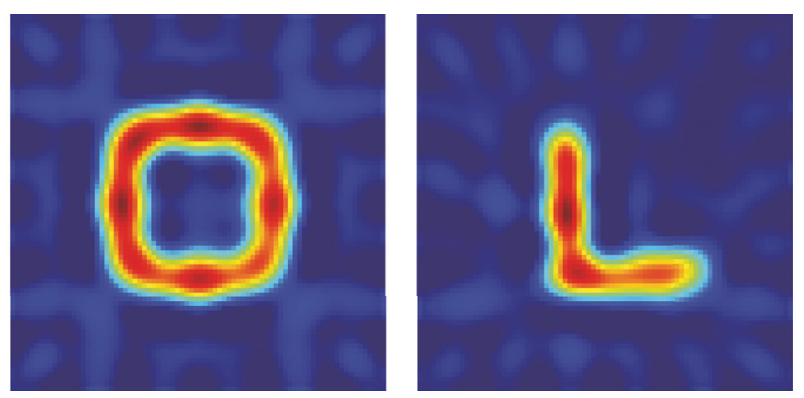

(c)

FIGURE 5: Simulated images with different shape. (a) shows three targets with different shape. (b) shows the images reconstructed by SRTI. (c) shows the images restored by our proposed method.

\section{Results and Discussion}

This section presents images that are restored using the reconstruction and restoration techniques described in Section 2. The images contain both simulated and experimental results, and the imaging accuracy of targets is also presented.

4.1. Simulated Results. This simulation part is used to evaluate the estimation method and the restoration method at ideal conditions with no noise. The simulated RTI system contains 20 wireless sensor nodes, and each node is placed $1.6 \mathrm{~m}$ apart along the perimeter of $8 \times 8 \mathrm{~m}^{2}$. The simulated targets are three types: rectangle, L-shape, and hollow block, as shown in Figure 5(a). The data of the simulations is obtained by the model described in (4), but without any noise. Then we use the SRTI reconstruction and image restoration techniques to obtain images, and Table 1 lists the SRTI model and image restoration parameters used in simulation phase.

Figure 5 shows the images of SRTI reconstructed result and restored result in different target types. We can find that the restored edge of targets is more distinguishable than SRTI reconstruction, and the image area of targets also converges to the middle line of targets. The corners of the L-type and hollow block are restored more accurately than SRTI reconstruction. We introduce Edge Preservation Index (EPI) [24] and Structural SIMilarity (SSIM) [25] to assess the improvement of our proposed method. The EPI and SSIM values of three targets are listed in Table 2.

$$
\begin{aligned}
\mathrm{EPI} & =\frac{\sum\left[\left|\Delta_{x} I_{c}(x, y)\right|+\left|\Delta_{y} I_{c}(x, y)\right|\right]}{\sum\left[\left|\Delta_{x} I_{o}(x, y)\right|+\left|\Delta_{y} I_{o}(x, y)\right|\right]}, \\
\mathrm{SSIM} & =\frac{1}{R} \sum_{R} \frac{\left(2 \mu_{c} \mu_{o}+C_{1}\right)\left(2 \sigma_{c o}+C_{2}\right)}{\left(\mu_{c}^{2}+\mu_{o}^{2}+C_{1}\right)\left(\sigma_{c}^{2}+\sigma_{o}^{2}+C_{2}\right)},
\end{aligned}
$$

where $\Delta_{x} I(x, y)=I(x, y)-I(x+1, y)$ and $\Delta_{y} I(x, y)=$ $I(x, y)-I(x, y+1)$ describe the differences of the image $I(x, y)$ on horizontal and vertical directions, respectively. $I_{c}(x, y)$ is the compared image, and $I_{o}(x, y)$ is the simulated original image. In (25), $\mu_{c}, \mu_{o}$ and $\sigma_{c}, \sigma_{o}$ are the means and 
TABLE 1: Parameters setting.

\begin{tabular}{lccc}
\hline Para. & Value (simulation) & Value (experiment) & Description \\
\hline$\alpha$ & 50 & 100 & Regularization parameter \\
$\lambda$ & 0.05 & 0.05 & Width of weighting ellipse (m) \\
$\beta$ & 0.01 & 0.01 & Learning rate \\
$\gamma$ & 0.05 & 0.08 & Smooth parameter \\
$K$ & 15 & 17 & Degradation function length \\
\hline
\end{tabular}

TABLE 2: EPI and SSIM comparison.

\begin{tabular}{|c|c|c|c|c|c|c|}
\hline \multirow[b]{2}{*}{ Target type } & \multicolumn{3}{|c|}{ EPI } & \multicolumn{3}{|c|}{ SSIM } \\
\hline & SRTI & Proposed & Improved & SRTI & Proposed & Improved \\
\hline Rectangle & 0.5859 & 0.6970 & $19.96 \%$ & 0.3679 & 0.4503 & $22.40 \%$ \\
\hline Hollow block & 0.5420 & 0.6614 & $22.3 \%$ & 0.1347 & 0.2085 & $54.79 \%$ \\
\hline L-shape & 0.6739 & 0.7345 & $8.99 \%$ & 0.4526 & 0.5433 & $20.04 \%$ \\
\hline
\end{tabular}

standard deviations of the images being compared and $\sigma_{c o}$ is the covariance between two images. These quantities are calculated in a local window which is centered on each pixel, and $R$ is the number of local windows in the image. $C_{1}$ and $\mathrm{C}_{2}$ are constants.

From Table 2, we note that the EPI and SSIM values from our proposed method of different target types are both larger than SRTI due to the decreased dilation effect in restored images. For "hollow block," the EPI and SSIM values from SRTI reconstructed image have the minimal values, and our proposed method has $22.3 \%$ and $54.79 \%$ increase compared to SRTI, respectively. The SSIM values of "hollow block" from both methods are much lower than the other two target types. This is due to the fact that the RSS values from limited wireless links cannot reconstruct the fine structure of the target. Overall, our proposed method restores more convergent and accurate edges of the targets than SRTI.

4.2. Experimental Results. The experimental RTI system contains 20 TI CC2530 wireless sensor nodes, and each node is placed $1.9 \mathrm{~m}$ apart along the perimeter of $9.5 \times 9.5 \mathrm{~m}^{2}$, as shown in Figure 6. The nodes operate at $2.4 \mathrm{GHz}$ ISM frequency band, and we use the token ring protocol to obtain RSS of the links in the real time [11]. Human target is set in the surveillance area. Then we use the reconstruction and restoration techniques to obtain images, and Table 1 lists the SRTI model and image restoration parameters used in the experimental phase.

Figure 7 shows the images when there are human targets within the surveillance area. Figures 7 (a) and 7(b) show the SRTI reconstructed image and the corresponding restored image when there is one person within the surveillance area. Figures 7(c) and 7(d) show the SRTI reconstructed image and the corresponding restored image when there are two persons within the surveillance area. Figure 7(c) illustrates the streaks of two individual targets interweaved with each other, and it is difficult to separate. Our proposed restoration method could isolate the targets easily, as shown in Figure $7(\mathrm{~d})$. Comparing the images, we can conclude that our proposed RTI restoration method improves the quality of imaging in these aspects: the target area is more convergent, the target area is isolated from the streaks, and the edge shape is more distinguishable.

We introduce image entropy to provide a quantitative assessment of the imaging performance [26]. This entropy is higher when the image has a poorer resolution.

$$
\begin{aligned}
E & =-\sum_{n=1}^{N} s s\left(x_{n}, y_{n}\right) \ln \left[s s\left(x_{n}, y_{n}\right)\right], \\
s s\left(x_{n}, y_{n}\right) & =\frac{\left|I\left(x_{n}, y_{n}\right)\right|^{2}}{\sum_{n=1}^{N}\left|I\left(x_{n}, y_{n}\right)\right|^{2}},
\end{aligned}
$$

where $I\left(x_{n}, y_{n}\right)$ represents the pixel value at position $\left(x_{n}, y_{n}\right)$ and $N$ is the total number of pixels in the image. The image entropy comparisons of different frames are shown in Figure 8. The image entropy from our proposed method has a lower value in each frame than SRTI, so we could get the same conclusions with the previous visual comparisons.

In order to assess the accuracy of SRTI and restored images, the "true" attenuation field should be known or estimated. Since the attenuation of the human body is difficult to model, we employ the simply uniformly attenuating cylinder to model a human body plane [7]. Now we can define the "true" image $\mathbf{x}_{o}$ of a human located at the point $c_{h}$ :

$$
x_{o n}= \begin{cases}1 & \text { if }\left\|x_{n}-c_{h}\right\|<R_{h} \\ 0 & \text { otherwise, }\end{cases}
$$

where $x_{n}$ is the position of the $n$th voxel in $\mathbf{x}_{o}$ and $x_{o n}$ is its value.

Then we obtain the normalized image $\widehat{\mathbf{f}}_{N}$ by scaling the estimated image (11), and we can define the mean-squared error (MSE) [27] of the normalized image as

$$
\mathrm{MSE}=\frac{\left\|\mathbf{x}_{o}-\widehat{\mathbf{f}}_{N}\right\|^{2}}{N},
$$

where $N$ is the number of voxels in the image. 


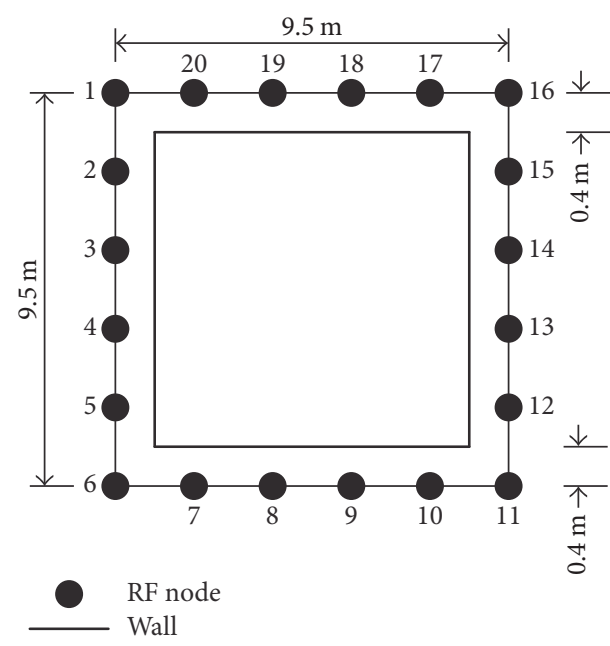

(a)

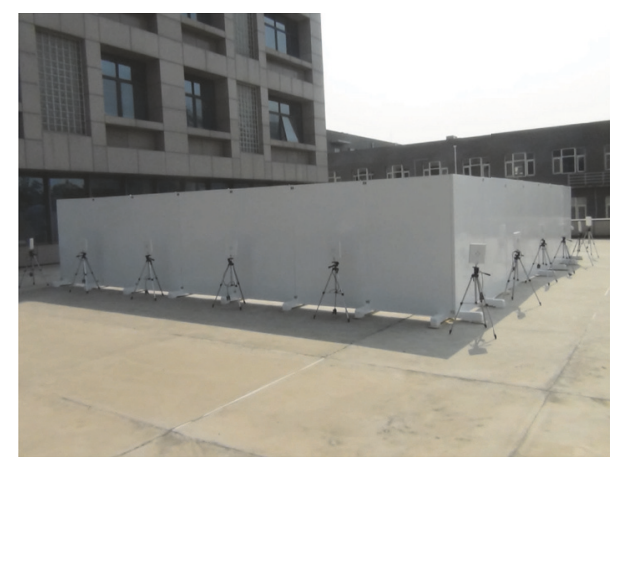

(b)

FIgure 6: The experimental environment. (a) The network geometry. (b) Photograph of the deployed network.

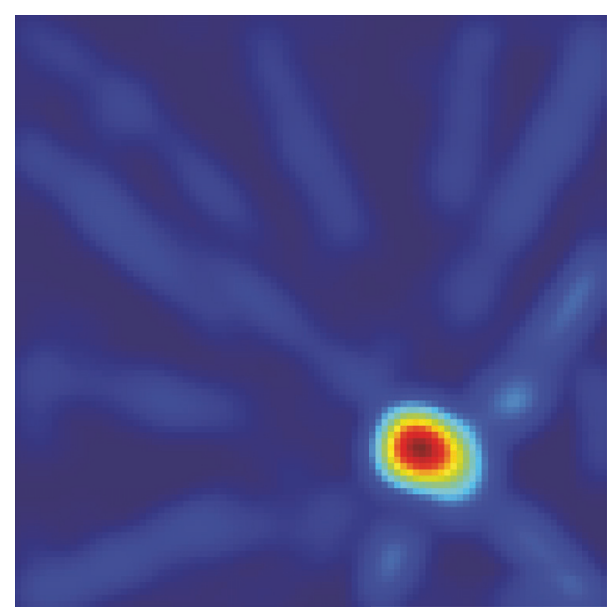

(a)

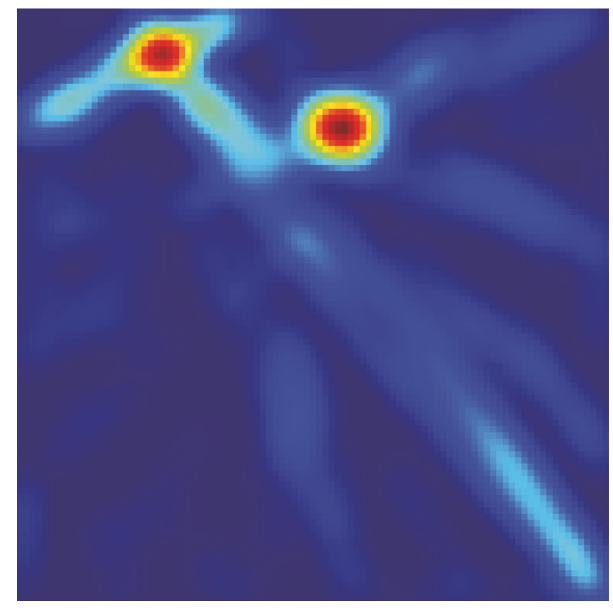

(c)

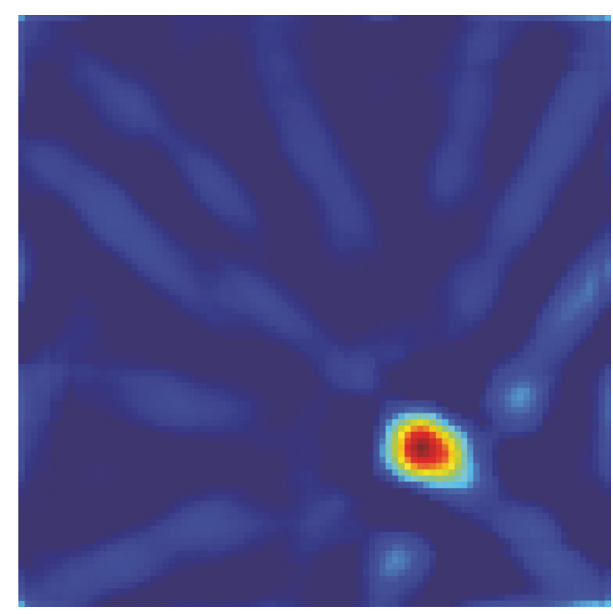

(b)

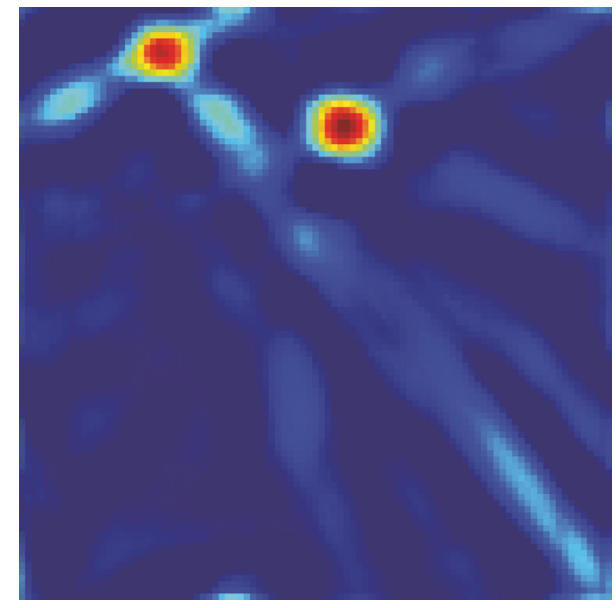

(d)

FIGURE 7: Visual comparison. (a) and (b) are the reconstructed and restored images when one person is within network area. (c) and (d) are the corresponding images when two persons are within network area. 


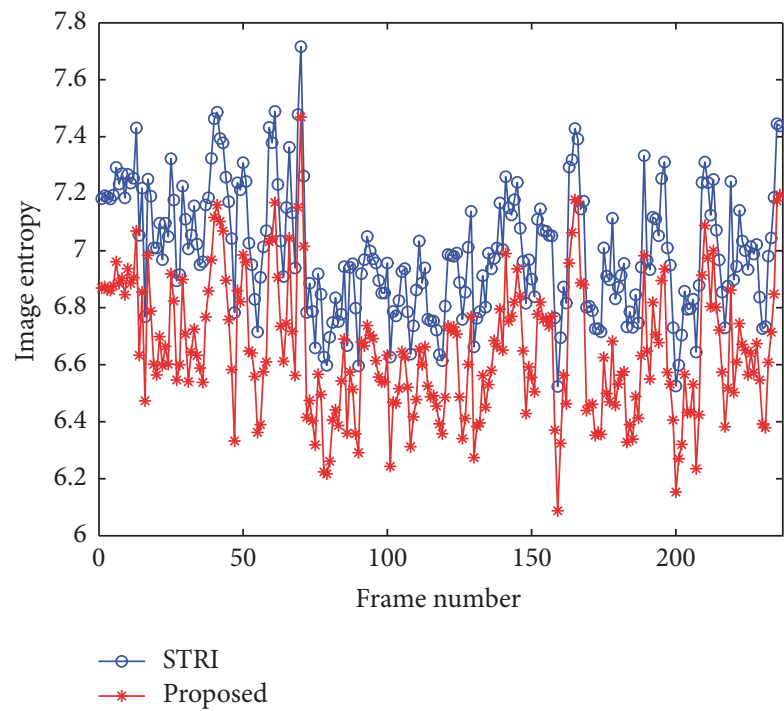

(a)

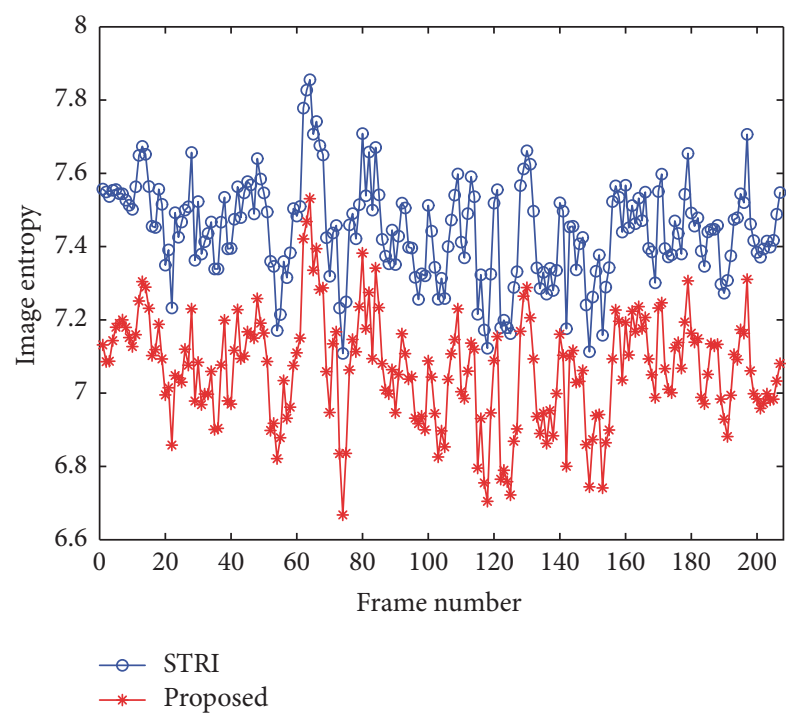

(b)

FIGURE 8: The image entropy comparisons of different frames. (a) is the image entropy comparison when only one person is within network area. (b) is the image entropy comparison when two persons are within network area.

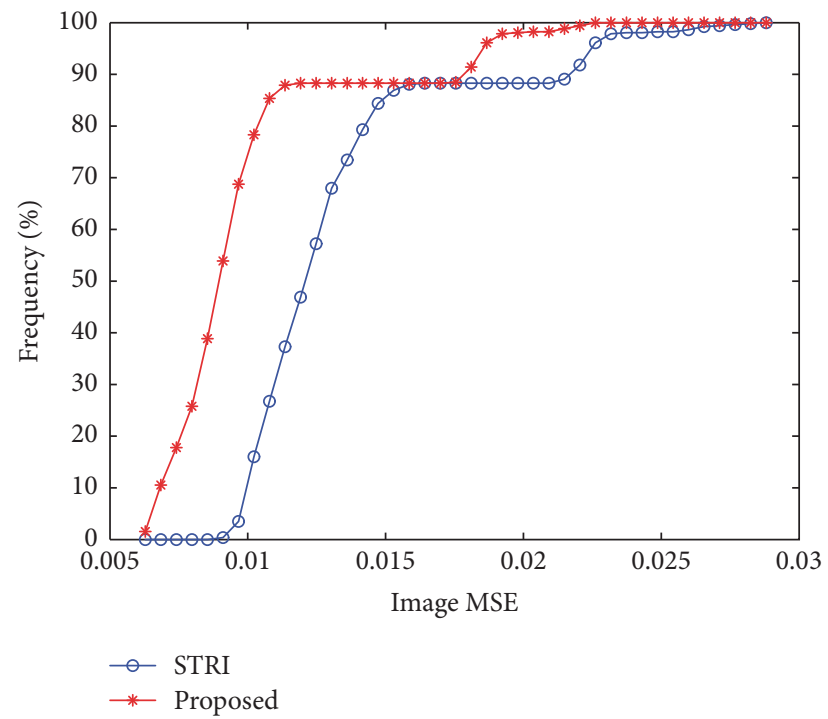

FIGURE 9: The image MSE comparison of shadowing-based RTI and proposed method.

In this experiment, we select nine different positions within the surveillance area, and a person stands for a few minutes on each position. Then we calculate MSE of the images after the SRTI reconstruction procedure and image restoration procedure, respectively. The cumulative distribution functions (CDFs) of image MSE for the RTI reconstruction and the proposed image restoration method are shown in Figure 9. When the radius of human model is set to $0.4 \mathrm{~m}$, the mean of image MSEs in different positions is 0.0135 with the SRTI reconstruction method and 0.0101 with the proposed image restoration method. Therefore, the proposed restoration method exhibits a preferable imaging accuracy.

\section{Conclusions}

In this paper, we introduce an image restoration technique to RTI system, and we present a novel model to estimate the degradation function of RTI system. The improvements concern three aspects: modeling the RTI reconstruction as the image degradation procedure, proposing an estimation method of degradation function of RTI system, and taking into consideration the inherent relationship of the linear transformation matrix.

The performance of the proposed method is validated in simulations and experiments. The results demonstrate that the proposed image restoration method outperforms the original shadowing-based RTI system. Moreover, the improvements both visually and numerically are positive, and the proposed method provides a novel solution for the accurate imaging of RTI system. The experiment results indicate the proposed method is capable of achieving a higher imaging accuracy.

Future research will investigate the other RTI reconstruction model that can reconstruct the fine geometrical shape of the target, and we will take the multipath components and noise interference into consideration.

\section{Conflicts of Interest}

The authors declare that there are no conflicts of interest regarding the publication of this paper.

\section{Acknowledgments}

This work was supported in part by National Natural Science Foundation of China (no. 61101129, no. 61227001, and no. 61471045) and Beijing Higher Education Young Elite Teacher Project (YETP1182). 


\section{References}

[1] M. Youssef, M. Mah, and A. Agrawala, "Challenges: device-free passive localization for wireless environments," in Proceedings of the 13th Annual ACM International Conference on Mobile Computing and Networking (MobiCom'07), pp. 222-229, Montréal, Québec, Canada, September 2007.

[2] C. R. Anderson, R. K. Martin, T. O. Walker, and R. W. Thomas, "Radio tomography for roadside surveillance," IEEE Journal on Selected Topics in Signal Processing, vol. 8, no. 1, pp. 66-79, 2014.

[3] B. Mager, N. Patwari, and M. Bocca, "Fall detection using RF sensor networks," in Proceedings of the IEEE 24th Annual International Symposium on Personal, Indoor, and Mobile Radio Communications, (PIMRC '13), pp. 3472-3477, London, UK, September 2013.

[4] J. Wilson and N. Patwari, "See-through walls: motion tracking using variance-based radio tomography networks," IEEE Transactions on Mobile Computing, vol. 10, no. 5, pp. 612-621, 2011.

[5] Y. Mostofi, "Cooperative wireless-based obstacle/object mapping and see-through capabilities in robotic networks," IEEE Transactions on Mobile Computing, vol. 12, no. 5, pp. 817-829, 2013.

[6] B. Beck, R. Baxley, and X. Ma, "Regularization techniques for floor plan estimation in radio tomographic imaging," in Proceedings of the 1st IEEE Global Conference on Signal and Information Processing, (GlobalSIP '13), pp. 177-180, Austin, TX, USA, December 2013.

[7] J. Wilson and N. Patwari, "Radio tomographic imaging with wireless networks," IEEE Transactions on Mobile Computing, vol. 9, no. 5, pp. 621-632, 2010.

[8] O. Kaltiokallio, M. Bocca, and N. Patwari, "A fade level-based spatial model for radio tomographic imaging," IEEE Transactions on Mobile Computing, vol. 13, no. 6, pp. 1159-1172, 2014.

[9] Y. Zhao, N. Patwari, J. M. Phillips, and S. Venkatasubramanian, "Radio tomographic imaging and tracking of stationary and moving people via kernel distance," in Proceedings of the 12th International Conference on Information Processing in Sensor Networks (IPSN '13), pp. 229-240, ACM, Philadelphia, PA, USA, April 2013.

[10] B. Wei, A. Varshney, N. Patwari, W. Hu, T. Voigt, and C. T. Chou, "DRTI: Directional radio tomographic imaging," in Proceedings of the 14th International Symposium on Information Processing in Sensor Networks, (IPSN '15), pp. 166-177, Seattle, Wash, USA, April 2015.

[11] Z. Wang, H. Liu, X. Ma, J. An, and S. Xu, "Enhancing indoor radio tomographic imaging based on interference link elimination," Digital Signal Processing: A Review Journal, vol. 44, no. 1, pp. 26-36, 2015.

[12] H. N. Manh, C. C. Huang, and L. Hsiao-Yi, "Landmark-based device calibration and region-based modeling for RSS-based localization," Wireless Communications and Mobile Computing, vol. 16, no. 13, pp. 1726-1745, 2016.

[13] J. Wang, R. V. Prasad, X. An, and I. G. M. M. Niemegeers, "A study on wireless sensor network based indoor positioning systems for context-aware applications," Wireless Communications and Mobile Computing, vol. 12, no. 1, pp. 53-70, 2012.

[14] F. Vanheel, J. Verhaevert, E. Laermans, I. Moerman, and P. Demeester, "Pseudo-3D RSSI-based WSN localization algorithm using linear regression," Wireless Communications and Mobile Computing, vol. 15, no. 9, pp. 1342-1354, 2015.

[15] R. C. Gonzalez and R. E. Woods, Digital Image Processing, Prentice Hall: Upper Saddle River, NJ, USA, 3rd edition, 2008.
[16] C. Sun, F. Gao, and H. Liu, "Super-resolution reconstruction of radio tomographic image," in Proceedings of the 83rd IEEE Vehicular Technology Conference, VTC Spring 2016, IEEE, Nanjing, China, May 2016.

[17] H. Liu, Z. Wang, X. Bu, and J. An, "Image reconstruction algorithms for radio tomographic imaging," in Proceedings of the IEEE International Conference on Cyber Technology in Automation, Control, and Intelligent Systems, (CYBER '12), pp. 48-53, Bangkok, Thailand, May 2012.

[18] J. Wilson, N. Patwari, and F. G. Vasquez, "Regularization methods for radio tomographic imaging," in Virginia Tech Symposium on Wireless Personal Communications, 2009.

[19] J. Jiang, X. Ma, C. Chen, T. Lu, Z. Wang, and J. Ma, “Single Image Super-Resolution via Locally Regularized Anchored Neighborhood Regression and Nonlocal Means," IEEE Transactions on Multimedia, vol. 19, no. 1, pp. 15-26, 2017.

[20] H. Takeda, S. Farsiu, and P. Milanfar, "Deblurring using regularized locally adaptive kernel regression," IEEE Transactions on Image Processing, vol. 17, no. 4, pp. 550-563, 2008.

[21] M. S. C. Almeida and M. A. T. Figueiredo, "Parameter estimation for blind and non-blind deblurring using residual whiteness measures," IEEE Transactions on Image Processing, vol. 22, no. 7, pp. 2751-2763, 2013.

[22] A. Rehman, M. Rostami, Z. Wang, D. Brunet, and E. R. Vrscay, "SSIM-inspired image restoration using sparse representation," Eurasip Journal on Advances in Signal Processing, vol. 2012, no. 1, article no. 16, 2012.

[23] A. Men, J. Xue, J. Liu, T. Xu, and Y. Zheng, "Applying background learning algorithms to radio tomographic imaging," in Proceedings of the 16th International Symposium on Wireless Personal Multimedia Communications, (WPMC '13), pp. 1-5, Atlantic City, NJ, USA, June 2013.

[24] C. Han, H. Guo, C. Wang, and D. Fan, "An enhanced sar image speckle filter," Journal of Remote Sensing-Beijing, vol. 8, no. 2, pp. 127-135, 2004.

[25] Z. Wang, A. C. Bovik, H. R. Sheikh, and E. P. Simoncelli, "Image quality assessment: from error visibility to structural similarity," IEEE Transactions on Image Processing, vol. 13, no. 4, pp. 600612, 2004.

[26] G. Gennarelli and F. Soldovieri, "Performance analysis of incoherent RF tomography using wireless sensor networks," IEEE Transactions on Geoscience and Remote Sensing, vol. 54, no. 5, pp. 2722-2732, 2016.

[27] H. Seddik, "A new family of Gaussian filters with adaptive lobe location and smoothing strength for efficient image restoration," Eurasip Journal on Advances in Signal Processing, vol. 2014, no. $25,2014$. 


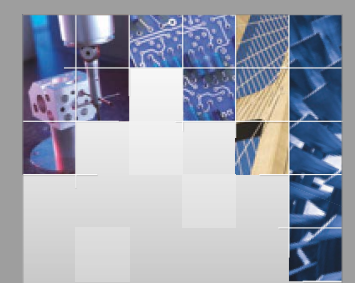

\section{Enfincering}
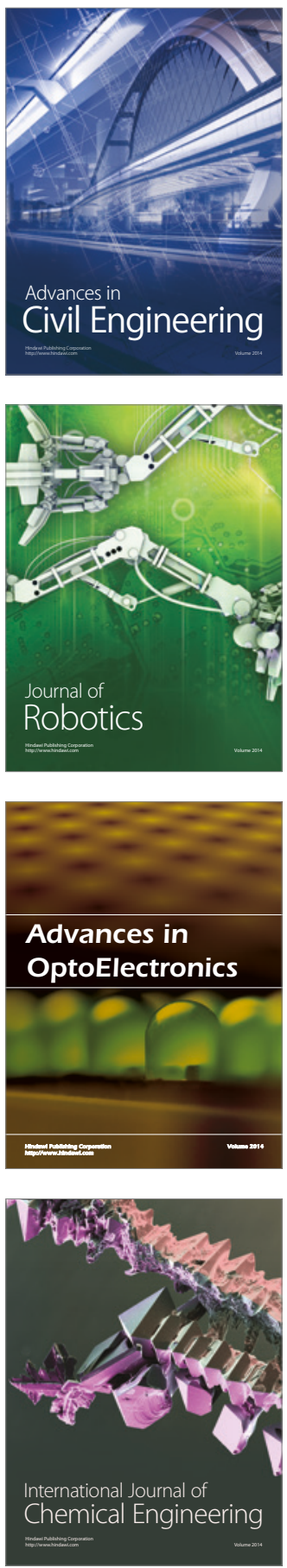

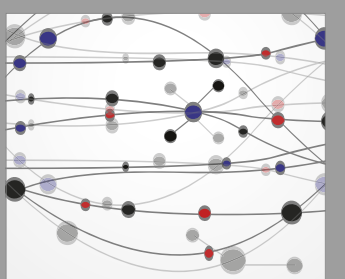

The Scientific World Journal

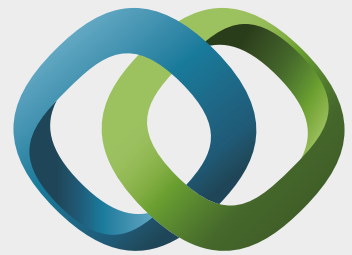

\section{Hindawi}

Submit your manuscripts at

https://www.hindawi.com
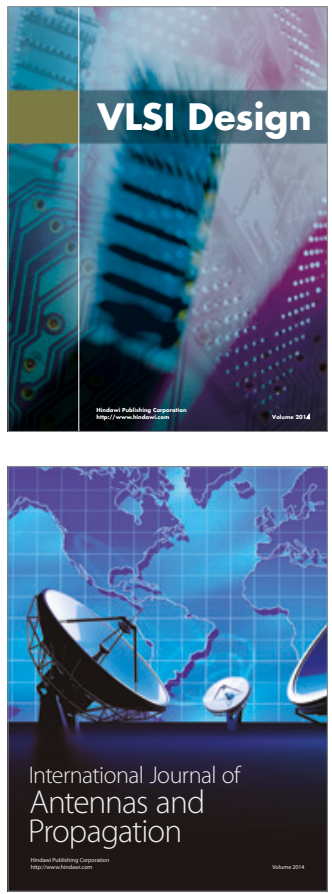

\section{Rotating}

Machinery
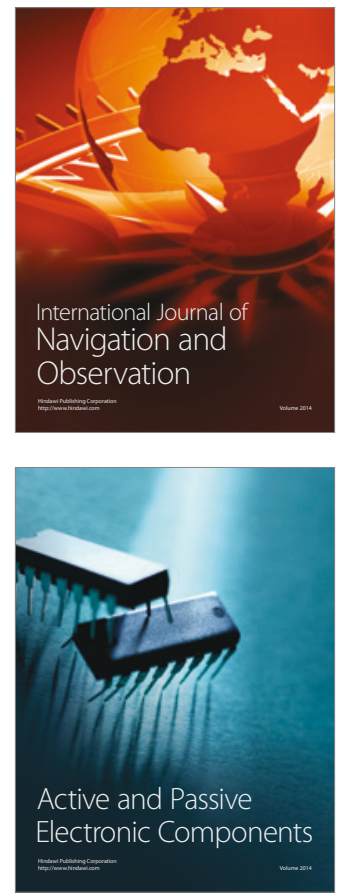
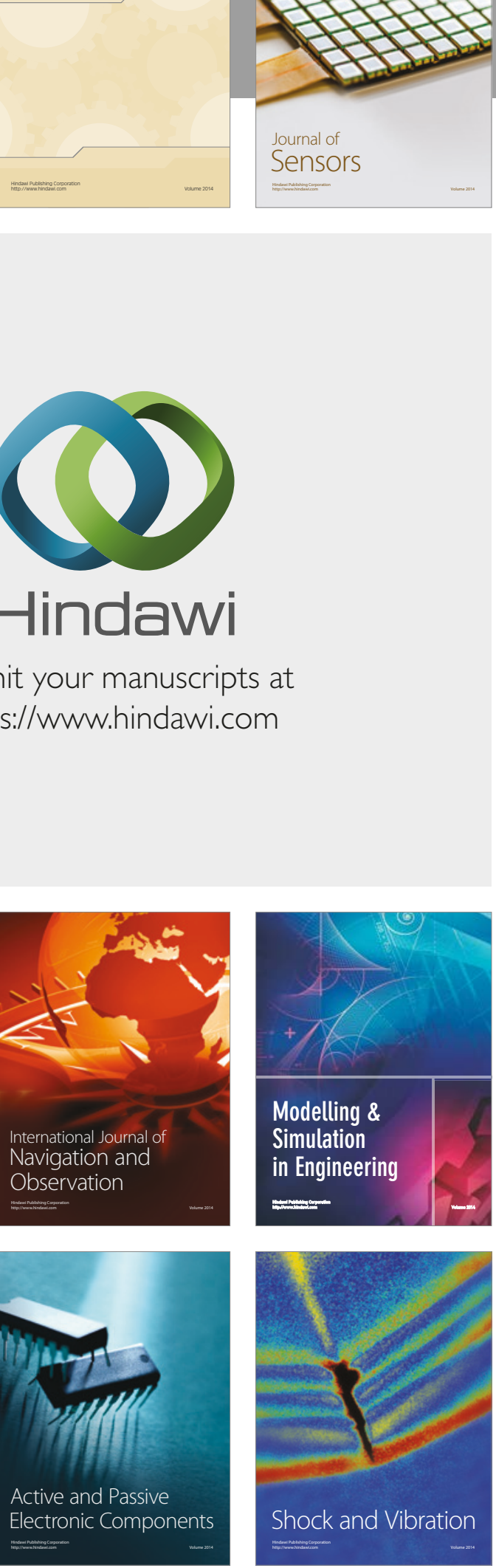
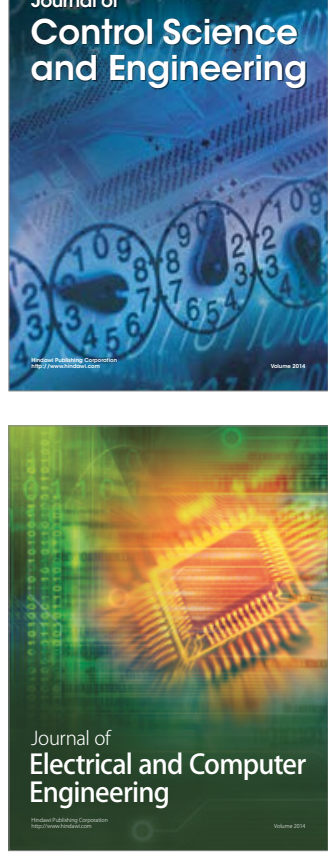

Distributed

Journal of

Control Science

and Engineering
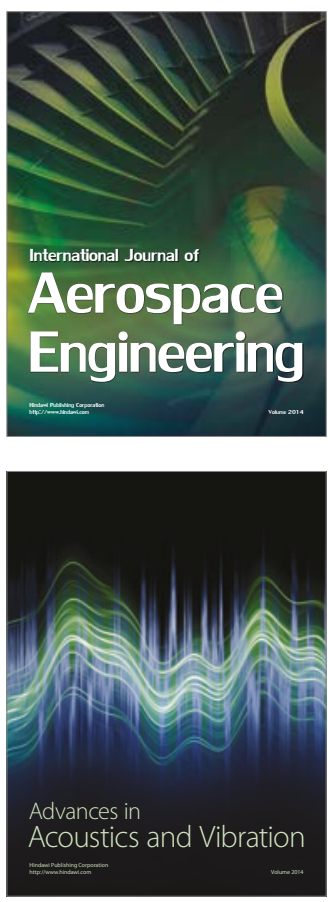

Sensor Networks 